Research article

\title{
Disassembly of the lens fiber cell nucleus to create a clear lens: The p27 descent
}

\author{
Sheldon Rowan ${ }^{\mathrm{a}, 1}$, Min-Lee Chang ${ }^{\mathrm{a}, 1}$, Natalie Reznikov ${ }^{\mathrm{b}}$, Allen Taylor ${ }^{\mathrm{a}, *}$ \\ a Tufts University JM-USDA Human Nutrition Research Center on Aging, Laboratory of Nutrition and Vision Research, 711 Washington Street Boston, MA, \\ 02111, USA \\ b Imperial College London, Depart of Materials, Prince Consort Road, South Kensington, London, SW7 2AZ, UK
}

\section{A R T I C L E I N F O}

\section{Article history:}

Received 24 November 2015

Received in revised form

11 February 2016

Accepted in revised form 29 February 2016

Available online 3 March 2016

\section{Keywords:}

Denucleation

Nuclear envelope

Lysosome

Mitosis

cdk1

Ubiquitin proteolytic system

Unfolded protein response

Cataract

\begin{abstract}
A B S T R A C T
The eye lens is unique among tissues: it is transparent, does not form tumors, and the majority of its cells degrade their organelles, including their cell nuclei. A mystery for over a century, there has been considerable recent progress in elucidating mechanisms of lens fiber cell denucleation (LFCD). In contrast to the disassembly and reassembly of the cell nucleus during mitosis, LFCD is a unidirectional process that culminates in destruction of the fiber cell nucleus. Whereas $\mathrm{p} 27^{\mathrm{Kip} 1}$, the cyclin-dependent kinase inhibitor, is upregulated during formation of LFC in the outermost cortex, in the inner cortex, in the nascent organelle free zone, p $27^{\mathrm{Kip} 1}$ is degraded, markedly activating cyclin-dependent kinase 1 (Cdk1). This process results in phosphorylation of nuclear Lamins, dissociation of the nuclear membrane, and entry of lysosomes that liberate DNaseII $\beta$ (DLAD) to cleave chromatin. Multiple cellular pathways, including the ubiquitin proteasome system and the unfolded protein response, converge on posttranslational regulation of $\mathrm{p} 27^{\mathrm{Kip}}$. Mutations that impair these pathways are associated with congenital cataracts and loss of LFCD. These findings highlight new regulatory nodes in the lens and suggest that we are close to understanding this fascinating terminal differentiation process. Such knowledge may offer a new means to confront proliferative diseases including cancer.
\end{abstract}

() 2017 Elsevier Ltd. All rights reserved.

\section{Introduction}

It is truly remarkable that using the same building blocks that are exploited to make other tissues that are opaque, nature also creates a clear accommodating lens that provides for our most precious sense, vision. Establishing a clear lens requires the removal of subcellular organelles and complete disintegration of the nucleus, a process referred to as lens fiber cell denucleation (LFCD). Outside of the lens, only two other types of cells denucleate; skin and red blood cells. But whereas in skin and red blood cells the nuclei are extruded from the cell, in the lens, LFCD involves intracellular disassembly of the nucleus in situ (Bassnett, 2009;

Abbreviations: LFCD, lens fiber cell denucleation; NE, nuclear envelope; UPS, ubiquitin proteolytic system; UPR, unfolded protein response; FIB-SEM, focused ion beam-scanning electron microscopy.

* Corresponding author.

E-mail addresses: sheldon.rowan@tufts.edu (S. Rowan), min-lee.chang@tufts. edu (M.-L. Chang), n.reznikov@imperial.ac.uk (N. Reznikov), allen.taylor@tufts.edu (A. Taylor).

1 These authors contributed equally.
Kuwabara and Imaizumi, 1974). How LFCD is controlled and accomplished has remained an enigma for over a century (Rabl, 1899), but the last few decades have allowed insights into this aspect of lens biology.

Biochemical, genetic, and cell biologic studies revealed that cortical fiber cells that are preparing for LFCD begin expressing the acidic nuclease DNAseII $\beta$ (also known as DLAD) in lysosomes, which accumulate around the nuclear envelope (NE) (Bassnett and Mataic, 1997; De Maria and Bassnett, 2007; Nakahara et al., 2007). The requirement for DNAseII $\beta$ in LFCD was demonstrated using knockout mice, which retain nuclear chromatin and are cataractous. Subsequently, the NE is disassembled and DNAseII $\beta$ enters the nucleus and degrades chromatin. The process of LFCD is not coupled to removal of other organelles such as mitochondria and may involve distinct cellular processes including autophagy (Costello et al., 2013).

\section{Relationship between mitosis and LFCD}

Molecular requirements for LFCD and associations between this unidirectional, terminal, biologic process and the cyclic processes of 
cell replication began with discovery of multiple Cyclins and Cyclindependent kinases (Cdk) in lens fiber cells, including the mitotic kinase Cdk1 (Gao et al., 1995, 1999; He et al., 1998). The presence of cell cycle machinery, and particularly Cdk1 activity, was unanticipated, since Cdk1 activity is rarely observed in postmitotic cells (Tommasi and Pfeifer, 1995). Along with Cdk1, Cdk inhibitors $\mathrm{p} 57^{\mathrm{Kip} 2}$ and $\mathrm{p} 27^{\mathrm{Kip} 1}$ (hereafter referred to as p57 and p27) are also expressed in mature lens fiber cells, where they are found associated with Cdks (Gao et al., 1999; Zhang et al., 1998).

We advanced the idea that LFCD co-opts the mitotic machinery by using multiple mouse models that exhibit congenital cataract and associated loss of LFCD. Three of these models (collagen IV mutant, DN-FGFR, and dnNCOA6) elicit an unfolded protein response (Lyu et al., 2016). We also generated mouse models in which the ubiquitin pathway was perturbed by expression of the ubiquitin variant K6W-Ub (which has lysine 6 replaced by tryptophan). They also form nuclear cataracts but do not exhibit an unfolded protein response (Caceres et al., 2010; Liu et al., 2015). These models consistently show a failure of Lamin A/C phosphorylation, a known Cdk1 phosphorylation target, and failed LFCD (compare Fig. 1A and B upper versus lower panels, and Fig. 1C left versus right panels).

To test directly whether Cdk1 itself is critical for LFCD, we first established that Cdk1 protein is detected in lens fiber cells by immunohistological methods. We found that Cdk1 is nuclear and cytoplasmic in the bow region, but gradually becomes more nuclear toward the presumptive organelle free zone toward the center of the lens (Fig. 2A) (Chaffee et al., 2014). Cdk1 becomes activated through dephosphorylation by members of the Cdc25 phosphatase proteins. Immunofluorescence detection of phospho-Cdk1 (inactive) shows that the inactive protein is exclusively cytoplasmic (Fig. 2A), consistent with the idea that Cdk1 is expressed and in a functionally active state in lens fiber cells that are poised for LFCD.

Next we genetically deleted Cdk1 specifically in the lens using Cre/loxP technology. Lens-specific Cdk1-knockout mice (MLR10; $C d k 1^{\mathrm{L} / \mathrm{L}}$ - simplified as $\left.C d k 1-/-\right)$ undergo normal lens differentiation and even removal of other organelles, however, they retain fiber cell nuclei (Fig. 2B) (Chaffee et al., 2014). Consistent with loss of Cdk1 activity, we also observe loss of Lamin A/C phosphorylation (Fig. 1B, bottom panel), whereas, in WT lenses, phospho-Lamin A/C is clearly observed (Fig. 1B, top panel). Interestingly, in Cdk1-/lenses, the usual rounding of nuclei that precedes LFCD does occur, suggesting that the role for Cdk1 in LFCD is downstream of other morphological changes (Fig. 2B).

Downstream of NE breakdown, Cdk1 phosphorylates NuMA (Nuclear Mitotic Apparatus), a nuclear protein that is essential for mitotic spindle assembly and maintenance. NuMA phosphorylation results in movement of NuMA from the cell membrane to the spindle poles, resulting in chromosome segregation (Kotak and Gonczy, 2013). Consistent with a proposed role for NuMA as a chromatin organizer (Radulescu and Cleveland, 2010), we found pNuMA distributed as multiple foci throughout the entire nuclei of early differentiating fiber cells in WT lenses (Fig. 2C panels A1,2). Strikingly, rather than coalescing to a few or to two prominent foci, as they do in mitosis, pNuMA coalesces to a single large focus in denucleating cells (Fig. 2C, panel A3). As expected, pNuMA was rarely observed in $C d k 1-/$ - lenses. Taken together, these observations confirm Cdk1 as an important NuMA kinase (Fig. 2C) (Chaffee et al., 2014).

The altered localization of pNuMA during LFCD mirrors characterized changes to the structure of chromatin, as visualized by DAPI staining. Consistent with an absence of pNuMA foci in Cdk1-/ - lenses, the nuclei remain larger and the chromatin remains heterogeneously spread or less focused throughout the nucleus (Fig. 2D, compare panes C and D versus panels A and B) (Chaffee et al., 2014).

\section{Integrated control of Cdk1 activity via p27 - role of ubiquitin pathway}

The absence of nuclei indicates that there is little opportunity to replace the very long lived lens cell proteins. This would seem to put the lens in considerable jeopardy in the face of lifelong oxidative or other environmental stress (Shang and Taylor, 2012; Weikel and Taylor, 2011) As part of our exploration into the lens ubiquitin proteolytic system (UPS), we documented functions for multiple components of the UPS in lens function and maintenance of protein quality, and we expressed a mutant ubiquitin against a WT ubiquitin background in two strains of mice (FVB) and a WT Bfsp2 background (Caceres et al., 2010; Dudek et al., 2005; Jahngen et al., 1986; Liu et al., 2015; Shang et al., 1999, 1997, 2001). K6W-Ub lenses showed a delayed developmental phenotype, including microphthalmia, accumulation of insoluble protein aggregates, delayed LFCD, and congenital cataract (Fig. 3A,B) (Caceres et al., 2010; Liu et al., 2015).

Overexpressing K6W-Ub in the lens affects proteolytic functions of the UPS and also alters other proteolytic systems. Thus, there is a significant accumulation of high mass (undegraded) ubiquitin conjugates in the K6W lens. This is consistent with proteolytic insufficiency, and accumulation of such high mass moieties leads to protein aggregation (Shang et al., 2005) and may in itself be cataractogenic (Caceres et al., 2010; Liu et al., 2015). K6W-Ub lenses also have dysregulated Connexins, with consequent accumulation of calcium and activation of calpain (Liu et al., 2015). Calpain is expressed and active during fiber cell differentiation (De Maria et al., 2009), and its dysregulation is another cataractogenic insult (David and Shearer, 1984; Giblin et al., 1984).

In addition to illuminating functions of specific lysines on ubiquitin with regard to UPS function in general, the lenses also showed stabilized p27, but not other Cdk inhibitors, including p57 (Fig. 3C). Stabilization of $\mathrm{p} 27$ was associated with the accumulation of high-mass ubiquitin conjugates, suggesting that K6W-Ub overexpression impeded the proteolysis of p27 (Caceres et al., 2010). We also confirmed that in the lens p27 is a potent inhibitor of Cdk1 activity, suggesting that LFCD defects acted on a mitotic-like checkpoint (Caceres et al., 2010). This was corroborated by loss of Lamin $\mathrm{A} / \mathrm{C}$ phosphorylation in K6W-Ub lenses (Fig. 1A). Taken together, these findings inform that whereas exit from cell cycle and formation of lens fiber cells requires upregulatiton of p27, proper LFCD requires a fully functional UPS to degrade p27. This reduction of p27 releases Cdk1 activity. Similar findings for an intact UPS in zebrafish lens development has also been reported (Imai et al., 2010).

\section{Integrated control of Cdk1 activity via p27 - role of unfolded protein response}

Recently, we examined several additional models in which differentiation in general and LFCD, specifically, are delayed or do not occur. These include mutant collagen IV ( Col IV), dominant negative fibroblast growth factor receptor (dnFGFR), and dominant negative nuclear receptor coactivator 6 (dnNCOA6) (Firtina et al., 2009; Reneker et al., 2011; Wang et al., 2010). Common to each of these models is the activation of endoplasmic reticulum stress due to expression of the transgenes leading to an unfolded protein response (UPR), and associated stabilization of BiP (Fig. 4A) (Lyu et al., 2016). In comparison, expression of K6W-Ub does not show a UPR. In all mutant mice, p27 levels were elevated relative to WT lenses (Figs. $3 \mathrm{C}$ and $4 \mathrm{~A}, \mathrm{~B}$ ). Cell based studies showed that when a UPR was elicited by chemical inducers of ER stress, including 

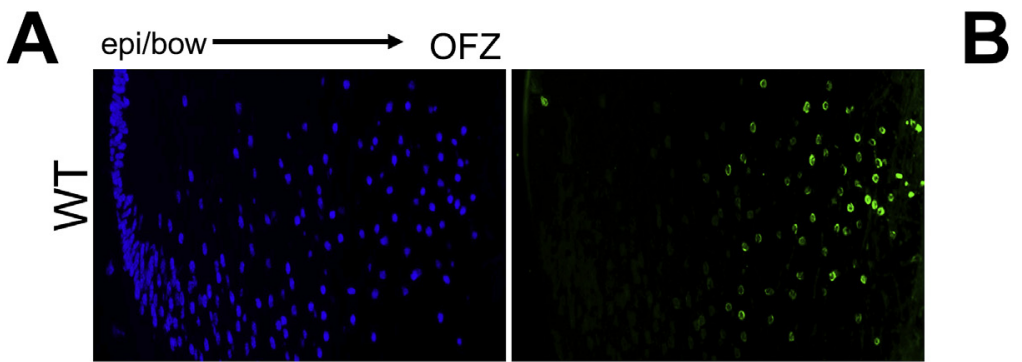

\section{pLaminA/C/DAPI}

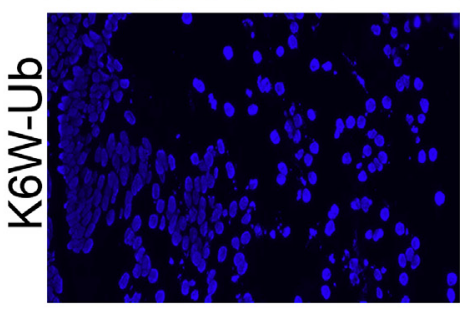

DAPI

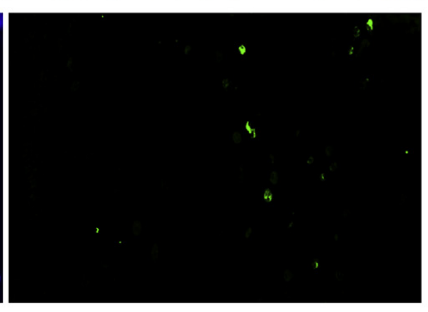

Phospho-Lamin A/C

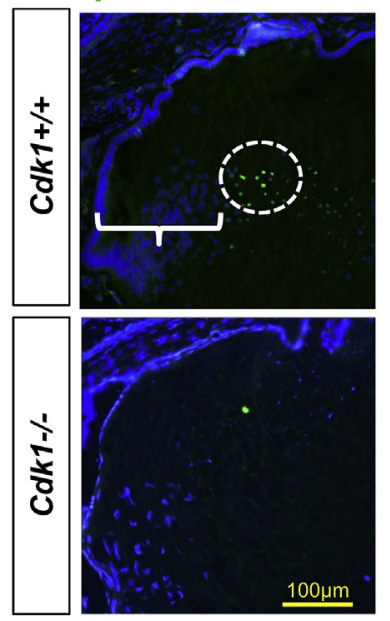

C WT Lens

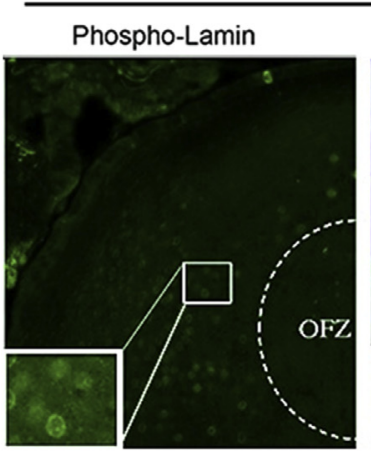

Phospho-Lamin + DAPI

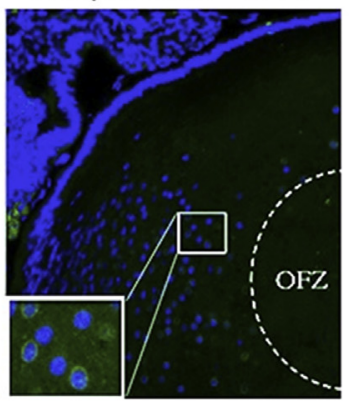

\section{Collagen IV lens}

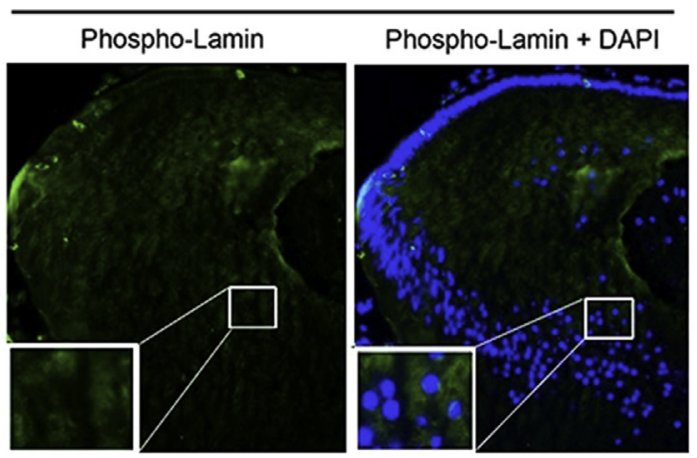

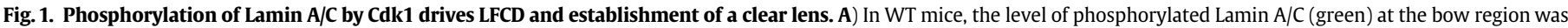

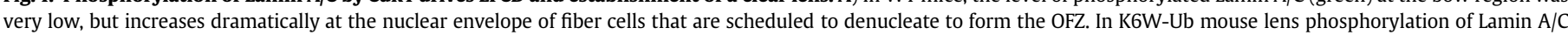

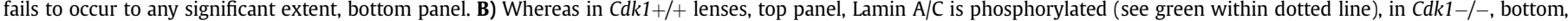

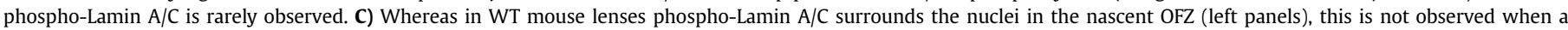
mutant of Collagen IV is expressed (right panels).

tunicamycin and thapsigargin treatment, p27 was also stabilized (Lyu et al., 2016). In contrast, limiting p27 did not affect BiP levels. In all cases, LFCD was delayed or abrogated, in association with loss of Lamin A/C phosphorylation (Fig. 1). Summarized, these findings indicate that multiple stress response pathways converge upon p27 stabilization, which serves as a common checkpoint in control of LFCD.

\section{Lysosomal entry downstream of Cdk1}

The activity and localization of DNaseII $\beta$ must be carefully controlled to preserve chromatin in non-denucleating cells. Using immunohistochemical localization, we find that in cells that do not undergo LFCD, either due to overexpression of K6W-Ub (Fig. 5A) or loss of Cdk1 (Fig. 5B) in the lens, DNaseII $\beta$ expression is not observed inside the nucleus; instead DNaseII $\beta$ is found surrounding the NE (Caceres et al., 2010; Chaffee et al., 2014). Thus, part of the function of the p27-Cdk1 checkpoint is to prevent nuclear entry of DNaseII $\beta$.

We utilized high-resolution focused ion beam - scanning electron microscopy (FIB-SEM) to visualize the status of lysosomes in lens fiber cells that have not undergone LFCD, or are activity engaged in denucleation. Intact fiber cells show a distinct clustering of lysosomes directly around the NE (Fig. 6A,B). In contrast, in cells undergoing LFCD, lysosomes can be visualized directly within the nuclear matrix, often in conjunction with degraded chromatin (Fig. 6C-E). This is consistent with delivery of DNaseII $\beta$ to degrade chromatin to complete LFCD. Strikingly, the nucleolus can be seen largely intact, detectable as faint speckling by DAPI staining, for several additional days within the disintegrating nucleus (Fig. $2 \mathrm{C}$ panel A3, 6C). FIB-SEM also suggests that nuclei that are about to undergo disassembly are often in close proximity to each other. Additional experiments are needed to corroborate this observation.

\section{Conclusions}

These data allow us to relate function of the ubiquitin proteolytic system, calpain proteolytic systems, the unfolded protein response, chromatin processing and lens differentiation in an effort to solve a puzzle that has perplexed biologists and physicians for over 100 years: how does the lens remove its nuclei. Here we present data that supports the hypothesis that in order to achieve LFCD and lens clarity the following sequence of events occurs: Stabilization of p27 enables dividing epithelia to withdraw from cell cycle, stop proliferating, and begin to differentiate into fibers in the outer cortex. In the inner cortex, p27 is destroyed by the UPS, 


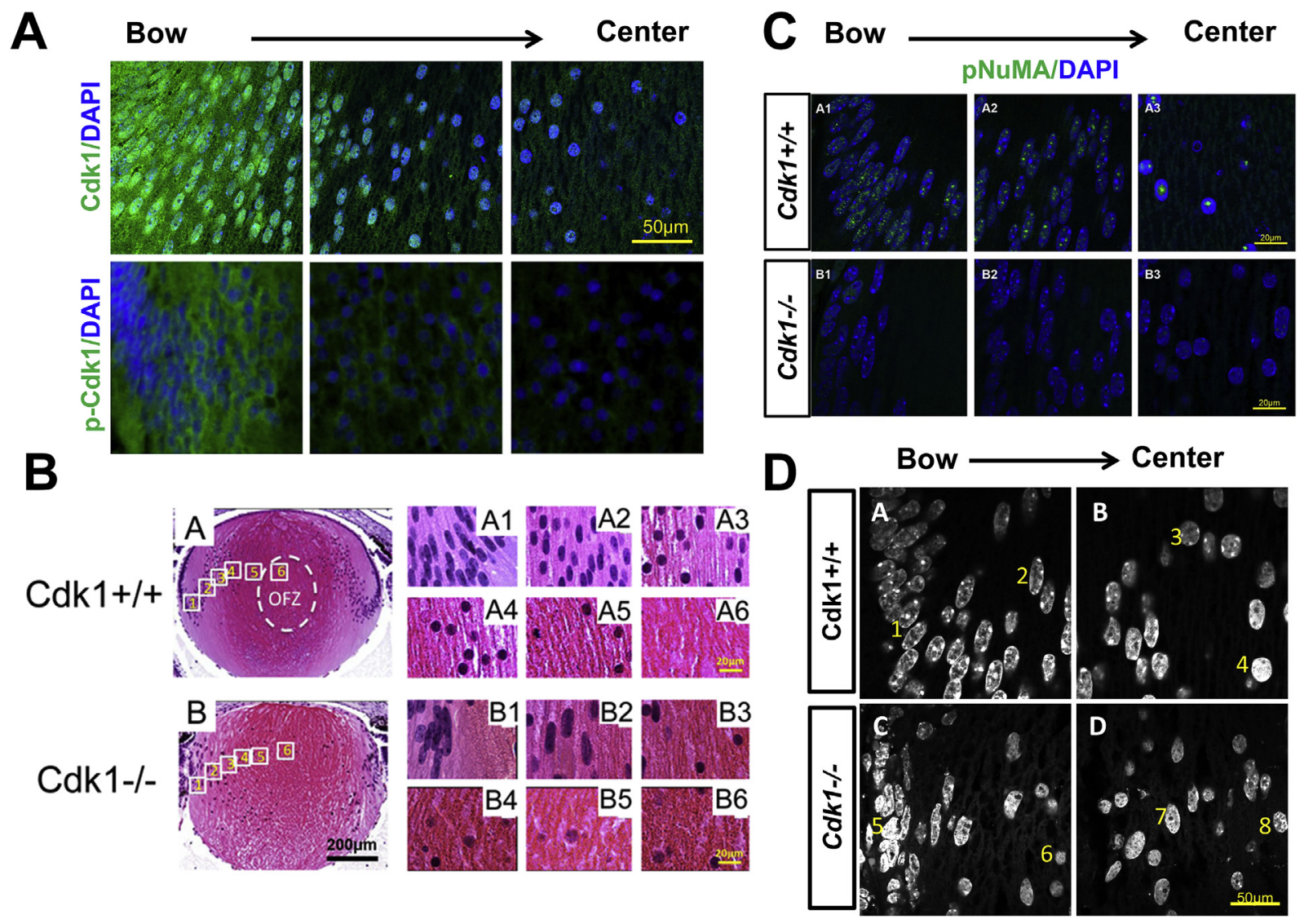

Fig. 2. Cdk1 becomes activated in the nucleus of fiber cells and is required for LFCD. Total Cdk1 is detected in both the nucleus and cytoplasm of fiber cells (A, top panels), whereas inactive phosphorylated Cdk1 is primarily cytoplasmic and at higher levels near the bow region in more immature fiber cells (A, bottom panels). WT mice undergo LFCD in the central part of the lens within the organelle free zone (OFZ) (B, panel A6). This is preceded by the elongation of nuclei of the outer fibers (B, panels A1-A2). In the interior of the lens the nuclei appear round (B, panels A3-A5) (B, panel A6). In contrast, Cdk1 deficient lenses retain nuclei even in the central lens (B, panel B6). Although elongated in the outer cortex, Cdk1-/- lens nuclei do go through the usual rounding process that precedes normal LFCD (B, compare B2 and B6). Phosphorylated NuMA is detected as numerous puncta throughout differentiating fiber cell nuclei (C, top panels A1 and A2), but is detected as a single punctum in denucleating cells (C, top panel A3). Phospho-NuMA is weakly expressed in Cdk1-l- lenses (C, bottom panels). WT fiber cell nuclei, stained by DAPI, show reproducible changes in chromatin organization from the outer fiber cells (D, panel A, nuclei 1,2) to inner fiber cells (D, panel B, nuclei 3,4). Cdk1-/- fiber cells do not show consistent chromatin states, with some outer fiber cells appearing poised to denucleate (D, panel C, nuclei $5,6)$ and some inner fiber cells resembling WT outer fiber cells (D, panel D, nuclei 7,8)
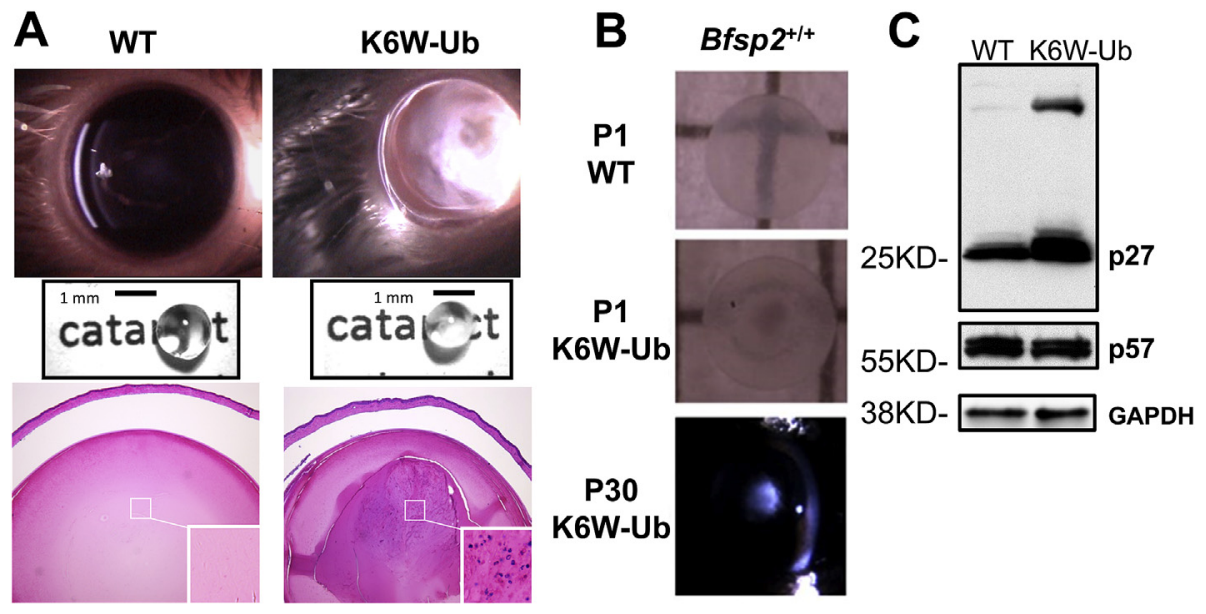

Fig. 3. K6W-Ubiquitin overexpression causes cataract, loss of LFCD, and stabilization of p27. Lenses overexpressing K6W-Ubiquitin (K6W-Ub) are microphthalmic, cataractous, and retain fiber cell nuclei in the center of the lens (A, compare right versus left panels). Cataracts are present at birth (B, middle panel) and are focused in the nucleus of the P30 K6W-Ub lens, even on a WT Bfsp2 background (B, bottom panel). p27 protein levels are increased in K6W-Ub P1 lenses, including some as distinct high-molecular weight bands, suggesting that some p27 is ubiquitinated but not degraded (C, top panel). p57 protein levels are unaltered in K6W-Ub lenses (C, middle panel), as are levels of the GAPDH loading control (C, bottom panel). 
A
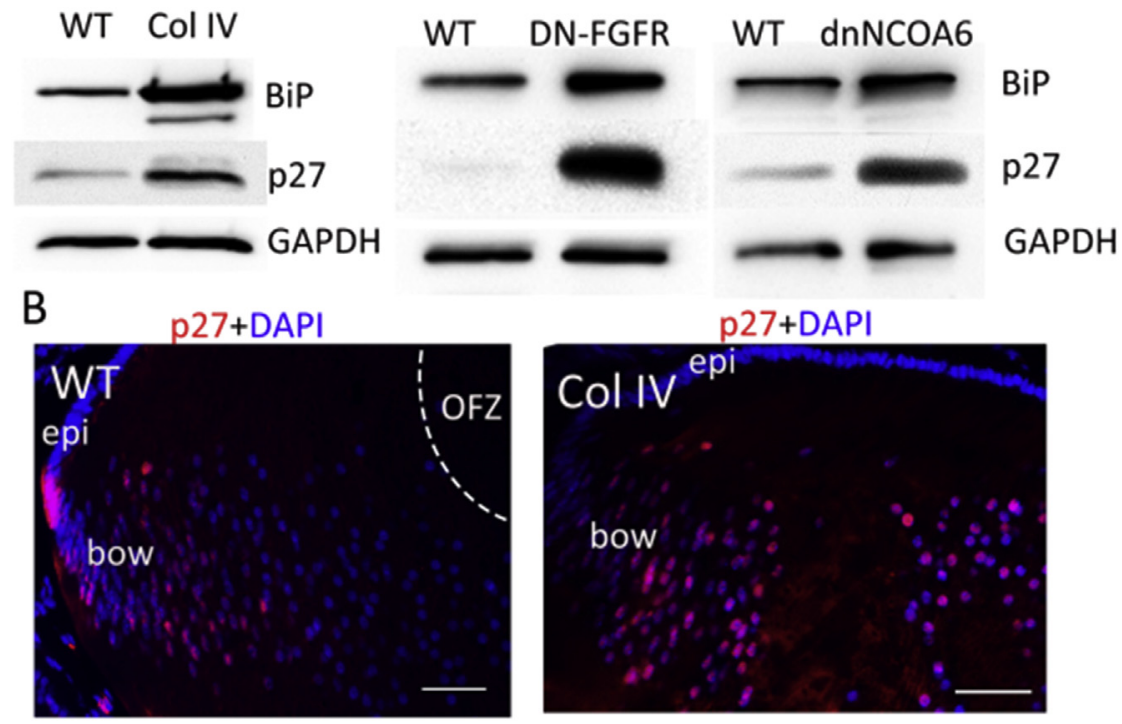

p27+DAPI

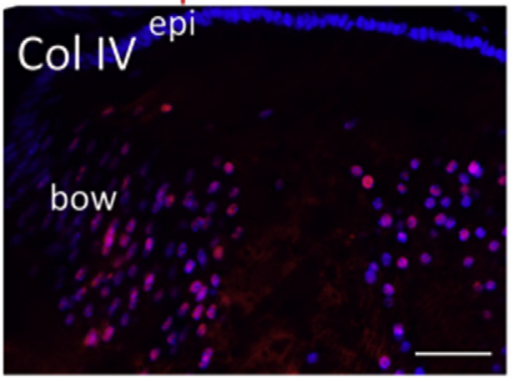

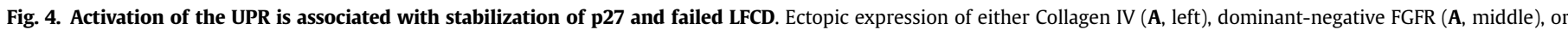

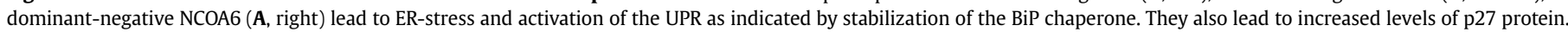

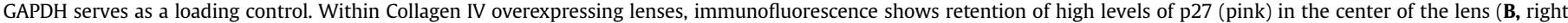
panel).

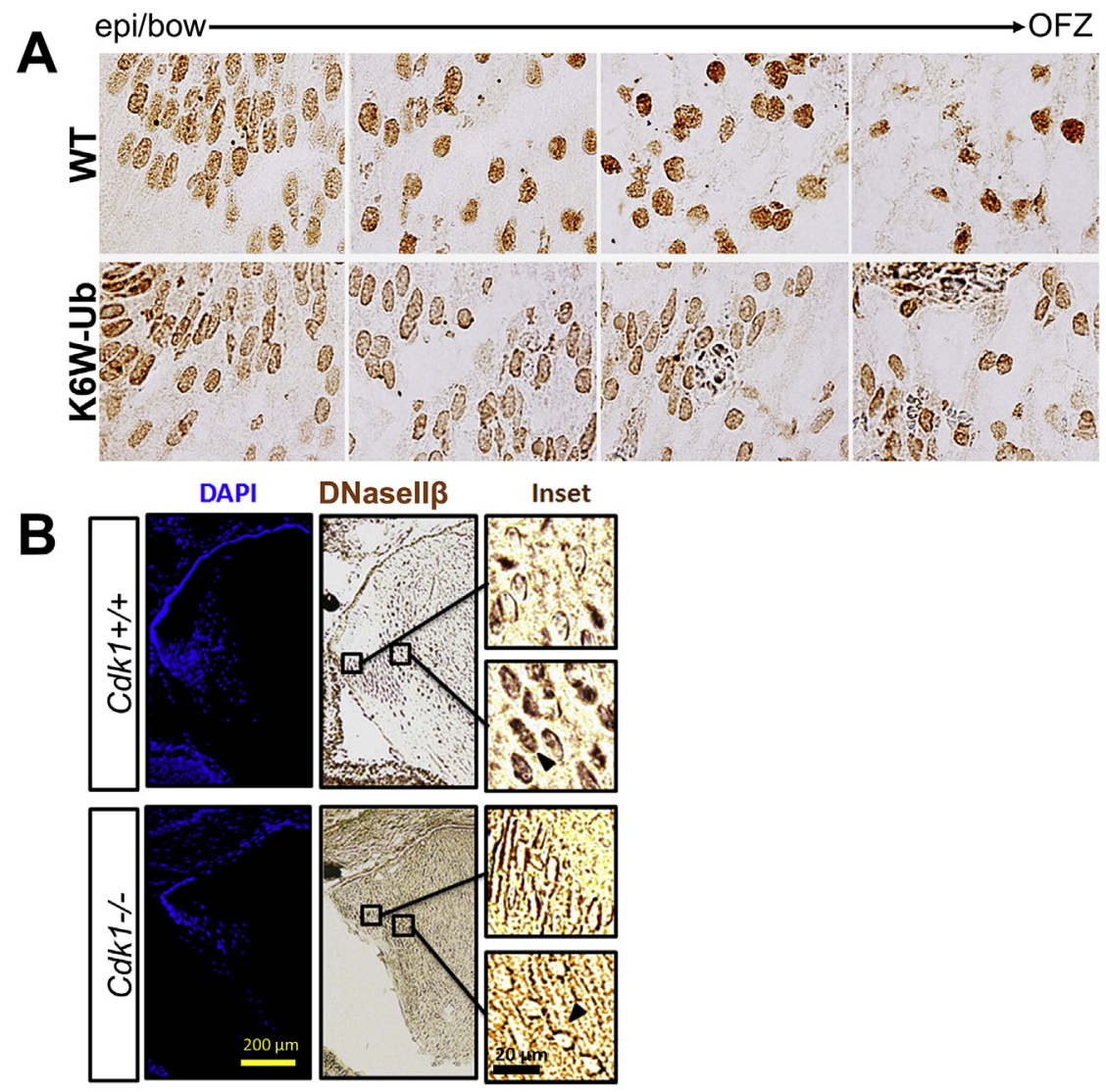

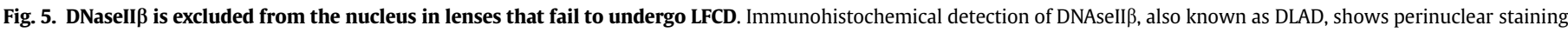

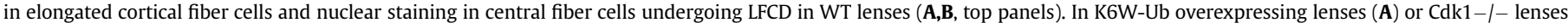
(B), DNaseII $\beta$ is expressed normally, but nuclear staining is markedly limited, even in the central lens (A,B, bottom panels).

Cdk1 localizes and is activated within nuclei. Nuclear lamins are phosphorylated, the nuclear envelope dissociates, chromatin is reorganized in a manner different from mitosis, lysosomes gain access to the nucleus and deliver DNaseII $\beta$ into the nucleoplasm. 

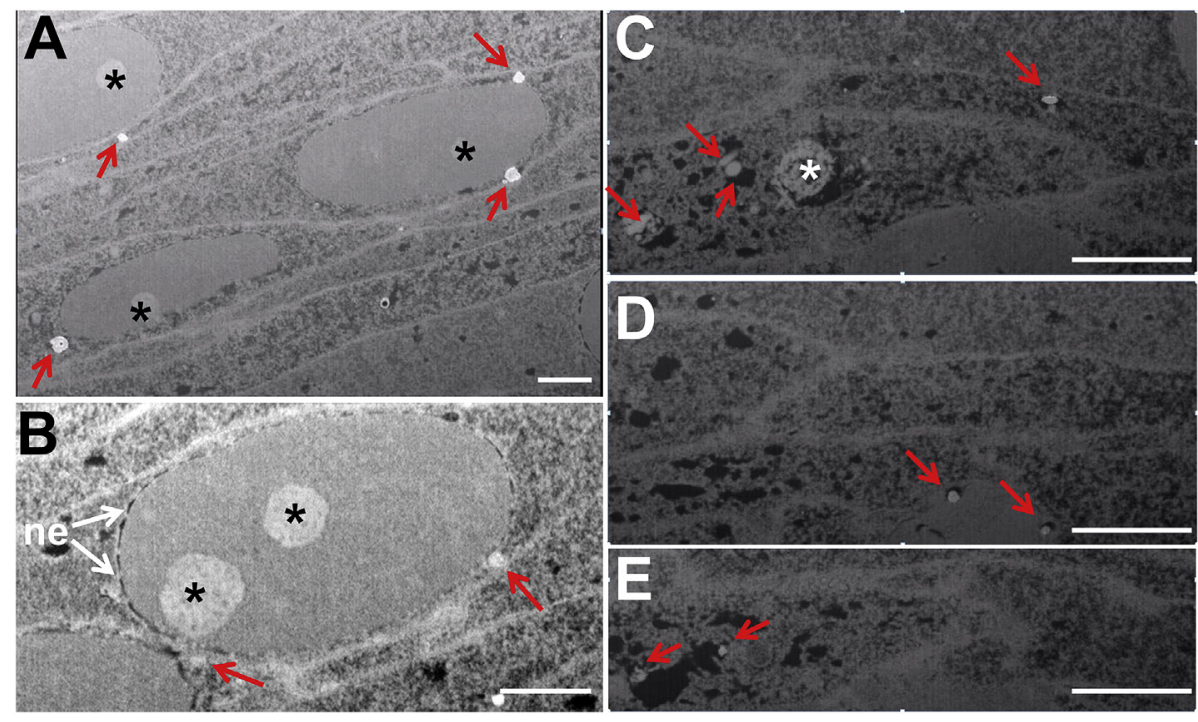

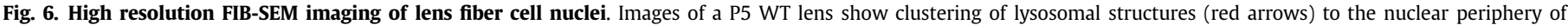

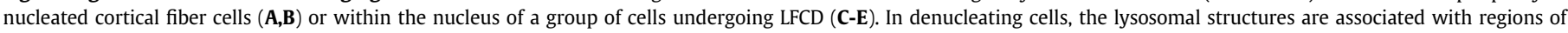

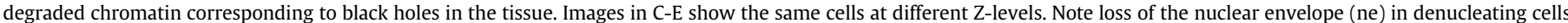

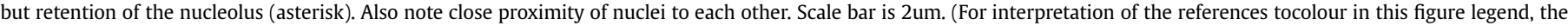
reader is referred to the web version of this article.)

Chromatin is degraded and the nuclear envelope is fully disassembled. Only then does the last trace of the nucleus, the nucleolus, finally disintegrate.

These findings address several critical aspects of lens biology. They demonstrate that Cdk1 is the primary kinase regulating LFCD. They also indicate that there is a pulse of p27 that directs LFC differentiation and p27 is the primary Cdk inhibitor involved in regulation of LFCD. Collectively, they corroborate the hypothesis that the unidirectional process of LFCD appropriates many events that drive the cyclical processes of cell proliferation. Various forms of proteolysis are implicated in lens development and the UPS and calpain systems are linked together for the first time. The UPS plays a critical role in LFCD in that many of the proteins that are involved in control of LFCD are substrates for the UPS (e.g. p27). While few roles of Lys 6 on ubiquitin are documented, it is clearly required for the progress of a proper differentiation program for lens development. Lys 6 is also required to avoid accumulation of abnormal and cytotoxic protein aggregates, some of which may be ubiquitin conjugates. The latter raises the possibility that unprocessed high mass ubiquitin conjugates may be cytotoxic. These large aggregates may also be cataractogenic. This hypothesis also suggests that cataractogenesis is related to other protein precipitation diseases such as Huntington 's disease, Alzheimer's disease, Parkinson's disease, etc ... We conclude that a fully functional UPS is required not only for cell proliferation of lens epithelium but also for the terminal differentiation of lens fiber cells, specifically removal of cell nuclei.

Whereas the UPS and Calpain proteolytic systems have been previously implicated in muscle wasting, a mechanism by which they interact has remained enigmatic (Smith et al., 2008). The observed interaction between the UPS and calpain proteolytic systems was a surprise. If confirmed in human lenses, it would explain why lenses liquefy in very aged people. It could also explain, in part, muscle atrophy in cases where there is dysregulated calcium homeostasis, such as in muscle wasting and diabetes.

\section{Future directions}

These findings beg several important questions, which will be investigated by our laboratory and others over the coming years:

1) What upstream events signal and initiate the destruction of p27 in order to facilitate normal LFCD? How is Cdk1 regulated?

2) What processes direct the lens fiber cell nucleus toward terminal destruction rather than reformation?

3 ) What governs the lens cortical fiber cell specific expression of DNaseII $\beta$ and how is DNaseII $\beta$ concentrated within lysosomes?

4) How do the lysosomes get targeted to the nucleus in advance of LFCD?

5) Do lysosomes enter the nucleus via a passive or regulated process and how does that relate to Cdk1-dependent changes to the NE?

6) Do lysosomes enter the nucleus intact or has lysosomal integrity been altered in preparation for- and in coordination with- LFCD, and if so, how? Are all components of the lysosome, including DNaseII $\beta$, simultaneously liberated into the nucleoplasm?

7) What is the role for other proteolytic enzymes including Cathepsins, Calpains, and Caspases (Wride, 2011) in the regulation and execution of LFCD?

8) Can we harness LFCD controls to arrest undesired proliferation such as occurs in cancer?

We anticipate that in order to answer these critical questions, multiple technologies and approaches will be required. These include development of novel mouse genetic models, especially those that address questions relating to the roles of p27 and factors upstream of Cdk1. Novel imaging will be required to visualize the process of LFCD, likely in conjunction with immunostaining. We believe that FIB-SEM is one such technology likely to emerge, since it has the ability to image deep into tissue and provide three dimensional reconstructions.

Currently, the single biggest missing piece in reconstructing LFCD is the ability to monitor the process in an in vitro mammalian system. Such a system will allow the use of pharmacological agents to test specific components of the process and will also allow live imaging of the process. This challenge is, in fact, secondary to the even larger challenge of following the process of full fiber cell maturation in a cell culture system; one that fully recapitulates cell 
cycle exit, fiber cell elongation, switches in intercellular communication systems, organelle removal, and finally LFCD.

\section{Acknowledgments}

This work is supported by the U.S. Department of Agriculture Agricultural Research Service (ARS) under agreement numbers 581950-0-014 and 58-1950-4-003 and by the NIH RO1EY13250, NIH RO1EY021212 to A.T.

\section{References}

Bassnett, S., 2009. On the mechanism of organelle degradation in the vertebrate lens. Exp. Eye Res. 88, 133-139.

Bassnett, S., Mataic, D., 1997. Chromatin degradation in differentiating fiber cells of the eye lens. J. Cell Biol. 137, 37-49.

Caceres, A., Shang, F., Wawrousek, E., Liu, Q., Avidan, O., Cvekl, A., Yang, Y, Haririnia, A., Storaska, A., Fushman, D., et al., 2010. Perturbing the ubiquitin pathway reveals how mitosis is hijacked to denucleate and regulate cell proliferation and differentiation in vivo. PLoS One 5, e13331.

Chaffee, B.R., Shang, F., Chang, M.L., Clement, T.M., Eddy, E.M., Wagner, B.D. Nakahara, M., Nagata, S., Robinson, M.L., Taylor, A., 2014. Nuclear removal during terminal lens fiber cell differentiation requires Cdk1 activity: appropriating mitosis-related nuclear disassembly. Development 141, 3388-3398.

Costello, M.J., Brennan, L.A., Basu, S., Chauss, D., Mohamed, A., Gilliland, K.O., Johnsen, S., Menko, A.S., Kantorow, M., 2013. Autophagy and mitophagy participate in ocular lens organelle degradation. Exp. Eye Res. 116, 141-150.

David, L.L., Shearer, T.R., 1984. Calcium-activated proteolysis in the lens nucleus during selenite cataractogenesis. Invest. Ophthalmol. Vis Sci. 25, 1275-1283.

De Maria, A., Bassnett, S., 2007. DNase II distribution and activity in the mouse lens. Investig. Ophthalmol. Vis. Sci. 48, 5638-5646.

De Maria, A.B., Shi, Y., Kumar, N.M., Bassnett, S., May 15 2009. Calpain expression and activity during lens fiber cell differentiation. J. Biol. Chem. 284 (20), 13542-13550.

Dudek, E.J., Shang, F., Valverde, P., Liu, Q., Hobbs, M., Taylor, A., 2005. Selectivity of the ubiquitin pathway for oxidatively modified proteins: relevance to protein precipitation diseases. Faseb J. 19.

Firtina, Z., Danysh, B.P., Bai, X., Gould, D.B., Kobayashi, T., Duncan, M.K., 2009. Abnormal expression of collagen IV in lens activates unfolded protein response resulting in cataract. J. Biol. Chem. 284, 35872-35884.

Gao, C.Y., Bassnett, S., Zelenka, P.S., 1995. Cyclin B, p34cdc2, and H1-kinase activity in terminally differentiating lens fiber cells. Dev. Biol. 169, 185-194.

Gao, C.Y., Rampalli, A.M., Cai, H.C., He, H.Y., Zelenka, P.S., 1999. Changes in cyclin dependent kinase expression and activity accompanying lens fiber cell differentiation. Exp. Eye Res. 69, 695-703.

Giblin, F.J., Hightower, K.R., Ragatzki, P.A., Reddy, V.N., 1984. Calcium-induced high molecular weight proteins in the intact rabbit lens. Exp. Eye Res. 39, 9-17.

He, H.Y., Gao, C., Vrensen, G., Zelenka, P., 1998. Transient activation of cyclin B/Cdc2 during terminal differentiation of lens fiber cells. Dev. Dyn. 211, 26-34.

Imai, F., Yoshizawa, A., Fujimori-Tonou, N., Kawakami, K., Masai, I., Oct 2010. The ubiquitin proteasome system is required for cell proliferation of the lens epithelium and for differentiation of lens fiber cells in zebrafish. Development 137 (19), 3257-3268.

Jahngen, J.H., Haas, A.L., Ciechanover, A., Blondin, J., Eisenhauer, D., Taylor, A., 1986.
The eye lens has an active ubiquitin-protein conjugation system. J. Biol. Chem. 261, 13760-13767.

Kotak, S., Gonczy, P., 2013. Mechanisms of spindle positioning: cortical force generators in the limelight. Curr. Opin. Cell Biol. 25, 741-748.

Kuwabara, T., Imaizumi, M., 1974. Denucleation process of the lens. Investig. Ophthalmol. 13, 973-981.

Liu, K., Lyu, L., Chin, D., Gao, J., Sun, X., Shang, F., Caceres, A., Chang, M.-L., Rowan, S., Peng, J., et al., 2015. Altered ubiquitin causes perturbed calcium homeostasis hyperactivation of calpain, dysregulated differentiation, and cataract. In: Proceedings of the National Academy of Sciences, 112, pp. 1071-1076.

Lyu, L., Whitcomb, E.A., Jiang, S., Chang, M.-L., Gu, Y., Duncan, M.K., Cvekl, A., Wang, W.-L., Limi, S., Reneker, L.W., et al., Mar 2016. Unfolded protein responseassociated stabilization of p27(Cdkn1b) interferes with lens fiber-cell denucleation leading to cataract. Faseb J. 30 (3), 1087-1095.

Nakahara, M., Nagasaka, A., Koike, M., Uchida, K., Kawane, K., Uchiyama, Y., Nagata, S., 2007. Degradation of nuclear DNA by DNase II-like acid DNase in cortical fiber cells of mouse eye lens. Febs J. 274, 3055-3064.

Rabl, C., 1899. Über den bau und die entwicklung der linse. III Teil: die lines der säugethiere. Z Wiss Zool. 67, 1-138.

Radulescu, A.E., Cleveland, D.W., 2010. NuMA after 30 years: the matrix revisited. Trends Cell Biol. 20, 214-222.

Reneker, L.W., Chen, H., Overbeek, P.A., 2011. Activation of unfolded protein response in transgenic mouse lenses. Investig. Ophthalmol. Vis. Sci. 52, 2100-2108.

Shang, F., Deng, G., Liu, Q., Guo, W., Haas, A.L., Crosas, B., Finley, D., Taylor, A., 2005 Lys6-modified ubiquitin inhibits ubiquitin-dependent protein degradation. J. Biol. Chem. 280, 20365-20374.

Shang, F., Gong, X., McAvoy, J.W., Chamberlain, C., Nowell Jr., T.R., Taylor, A., 1999 Ubiquitin-dependent pathway is up-regulated in differentiating lens cells. Exp. Eye Res. 68, 179-192.

Shang, F., Gong, X., Taylor, A., 1997. Activity of ubiquitin-dependent pathway in response to oxidative stress. Ubiquitin-activating enzyme is transiently upregulated. J. Biol. Chem. 272, 23086-23093.

Shang, F., Nowell Jr., T.R., Taylor, A., 2001. Removal of oxidatively damaged proteins from lens cells by the ubiquitin-proteasome pathway. Exp. Eye Res. 73, 229-238.

Shang, F., Taylor, A., 2012. Role of the ubiquitin-proteasome in protein quality control and signaling: implication in the pathogenesis of eye diseases. Prog. Mol. Biol. Transl. Sci. 109, 347-396.

Smith, I.J., Lecker, S.H., Hasselgren, P.O., 2008. Calpain activity and muscle wasting in sepsis. Am. J. Physiol. Endocrinol. Metab. 295, E762-771.

Tommasi, S., Pfeifer, G.P., 1995. In vivo structure of the human cdc2 promoter: release of a p130-E2F-4 complex from sequences immediately upstream of the transcription initiation site coincides with induction of cdc2 expression. Mol. Cell Biol. 15, 6901-6913.

Wang, W.L., Li, Q., Xu, J., Cvekl, A., 2010. Lens fiber cell differentiation and denucleation are disrupted through expression of the N-terminal nuclear receptor box of NCOA6 and result in p53-dependent and p53-independent apoptosis. Mol. Biol. Cell 21, 2453-2468.

Weikel, K., Taylor, A., 2011. Nutritional modulation of cataract and age-related macular degeneration. Mol. Asp. Med. 33, 318-375.

Wride, M.A., 2011. Lens fibre cell differentiation and organelle loss: many paths lead to clarity. Philos. Trans. R. Soc. Lond B Biol. Sci. 366, 1219-1233.

Zhang, P., Wong, C., DePinho, R.A., Harper, J.W., Elledge, S.J., 1998. Cooperation between the Cdk inhibitors p27(KIP1) and p57(KIP2) in the control of tissue growth and development. Genes Dev. 12, 3162-3167. 